\title{
Evaluación de la calidad del agua del río Cunas índices fisicoquimicos y biologicos, Junìn - Perú
}

\author{
Evaluation of the quality of the water of the river Cunas physiochemical and \\ biological indexes, Junín - Perú
}

\author{
María Custodio V. ', Fernán Chanamé Z. ' \& Wilfredo Bulege G. ${ }^{2}$ \\ ${ }^{1}$ Facultad de Zootecnia, Universidad Nacional del Centro del Perú. \\ ${ }^{2}$ Universidad Continental, Huancayo - Perú. \\ Email: custodiovillanueva65@yahoo.es
}

\section{RESUMEN}

Objetivo: Evaluar la calidad del agua del río Cunas mediante índices fisicoquímicos y biológicos a fin de identificar las zonas con buen estado de conservación, entre enero y diciembre de 2013. Métodos: Se utilizaron los métodos de observación, descripción y explicación, el diseño no experimental de tipo longitudinal. Se definieron tres sectores de muestreo, en Angasmayo (Concepción), Huarisca (Chupaca) y Pilcomayo (Huancayo). Las muestras de agua fueron colectadas en botellas de plástico de dos litros y en frascos de vidrio estériles para determinar la concentración de nitratos y fosfatos, y coliformes termotolerantes, respectivamente. Los parámetros medidos in situ fueron: oxígeno disuelto $(\mathrm{mg} / \mathrm{l})$, sólidos totales disueltos $(\mathrm{mg} / \mathrm{l})$, temperatura $\left({ }^{\circ} \mathrm{C}\right), \mathrm{pH}$ y turbidez (FTU). Estas mediciones se realizaron con equipos portátiles Hanna Instruments. Las muestras de macroinvertebrados bentónicos se colectaron mediante una red Surber con malla de $250 \mu \mathrm{m}$ de abertura. Resultados: según el INSF, Angasmayo presentó calidad de agua buena $(75,14$ en época de lluvia; 74,23 en estiaje), Huarisca calidad media (67,96 en Iluvia; 65,22, en estiaje) y Pilcomayo calidad media (61,39 en Iluvia; 55,82 en estiaje). Según el índice EPT, Angasmayo reveló buena calidad de agua, Huarisca regular calidad y Pilcomayo mala calidad de agua. Conclusiones: En Angasmayo, las masas de agua tanto en época de lluvia como en estiaje calificaron como agua de calidad buena. En Huarisca calificaron como agua de calidad media o regular y en Pilcomayo, como agua de calidad media (según INSF) y de calidad mala (según EPT).

\section{ABSTRACT}

Objective: To evaluate the quality of the water of the river Cunas physiochemical and biological indexes in order to identify the areas with good conservation state, between January of 2013 and December of 2013. Methods: The observation methods, description and explanation, the non-experimental design of longitudinal type were used. They were defined three sampling sectors, in Angasmayo (Concepción), Huarisca (Chupaca) and Pilcomayo (Huancayo). The samples of water were collected in bottles of plastic of two liters and in sterile glass flasks to determine the concentration of nitrates and phosphates, and coliformes termotolerantes, respectively. The parameters measured in situ were: dissolved oxygen $(\mathrm{mg} / \mathrm{l})$, dissolved total solids $(\mathrm{mg} / \mathrm{l})$, temperature $\left({ }^{\circ} \mathrm{C}\right)$, $\mathrm{pH}$ and turbidez (FTU). These mensurations were carried out with portable teams Hanna Instruments. The samples of benthic macroinvertebrates were collected by means of a net Surber with mesh of $250 \mu \mathrm{m}$ of opening. Results: according to the INSF, Angasmayo presented quality of good water (75,14 in rain time; 74,23 in low water), Huarisca half quality $(67,96$ in rain; 65,22, in low water) and Pilcomayo half quality $(61,39$ in rain; 55,82 in low water). According to the index EPT, Angasmayo revealed good quality of water, Huarisca to regulate quality and Pilcomayo bad quality of water. Conclusions: In Angasmayo, the masses of water so much in rain time as in low water they qualified like water of good quality. In Huarisca they qualified like water of half quality or to regulate and in Pilcomayo, like water of half quality (according to INSF) and of bad quality (according to EPT).

Palabras clave | Key words:

calidad de agua, río Cunas, índices.

quality of water, river Cradles, indexes. 


\section{INTRODUCCIÓN}

El agua es el recurso natural renovable que ejerce la acción más limitante en el desarrollo humano y el de toda forma de vida. Su disponibilidad siempre se había planteado desde la perspectiva de su abundancia, pero el progresivo descenso de su calidad en los últimos cincuenta años, ha dado lugar a considerables pérdidas económicas y ecológicas.

El vertimiento de afluentes domésticos e industriales y de sustancias peligrosas, como: agroquímicos, lixiviados provenientes de relaves abandonados de la minería y de botaderos de residuos sólidos a los cuerpos de agua genera la necesidad de aplicar un instrumento para dar seguimiento a la calidad de las aguas (1).

El uso de determinaciones fisicoquímicas ha sido el método clásico para evaluar la calidad del agua. Sin embargo, en la actualidad se han complementado con el método biológico mediante el uso de comunidades biológicas. Este método trabaja sobre la premisa que el nivel de respuesta de los organismos difiere según el tipo de contaminante a que han sido expuestos (2). En términos generales se puede decir que la biota acuática cambia su estructura y funcionamiento al modificarse las condiciones ambientales de sus hábitats naturales (3).

En el contexto de los acuerdos ambientales globales, el tema del agua resulta preponderante no sólo por su carácter estratégico sino por la preocupación que existe en torno al problema de su escasez y calidad, más aún cuando este problema es causado por factores antrópicos, los mismos que podemos evitar. De ahí que la calidad de agua potable, en especial, es un tema de preocupación y atención prioritaria para la Organización Mundial de la Salud porque el agua contaminada es un factor de múltiples enfermedades en la población (4).

En tal sentido, el problema general de investigación formulado fue: ¿Cuál es la calidad del agua del río Cunas evaluada mediante índices fisicoquímicos y biológicos? y como problemas específicos: (a) ¿̇Cuál es la calidad del agua evaluada mediante el índice de calidad de agua de la Fundación Nacional de Saneamiento de los Estados Unidos - INSF? (b) ¿Cuál es la calidad del agua evaluada mediante el índice biótico Ephemeroptera, Plecoptera y Trichoptera - EPT?

La hipótesis fundamental planteada fue: La Páginas 98-105 calidad del agua del río Cunas evaluada mediante índices fisicoquímicos y biológicos es buena en el sector de Angasmayo, es de calidad media en el sector de Huarisca y de calidad mala en el sector de Pilcomayo, en donde las presiones antrópicas son de diferente naturaleza.

Las hipótesis derivadas fueron: (a) La calidad del agua del río Cunas evaluada mediante el ICA - INSF es buena en el sector de Angasmayo, media en el sector de Huarisca y mala en el sector de Pilcomayo. (b) La calidad del agua del río Cunas evaluada mediante el índice biótico EPT es buena en el sector de Angasmayo, regular en el sector de Huarisca y mala en el sector de Pilcomayo.

Considerando los problemas e hipótesis planteadas se propuso como objetivo general: Evaluar la calidad del agua del río Cunas mediante índices fisicoquímicos y biológicos a fin de identificar las zonas con buen estado de conservación, entre enero y diciembre de 2013. Y como objetivos específicos: (a) Caracterizar la calidad del agua mediante el índice de calidad de agua ICA - NSF. (b) Caracterizar la calidad del agua mediante el índice biótico EPT.

\section{MATERIAL Y MÉTODOS}

\section{Descripción del área de estudio}

El área de estudio seleccionada se ubica en la subcuenca hidrográfica del río Cunas en la sierra central del Perú, sobre la margen derecha del río Mantaro. Está ubicada entre las coordenadas $11^{\circ} 45^{\prime}$ y $12^{\circ} 20^{\prime}$ latitud sur; $75^{\circ}$ $15^{\prime}$ y $75^{\circ} 45^{\prime}$ longitud oeste (ver figura 1). 


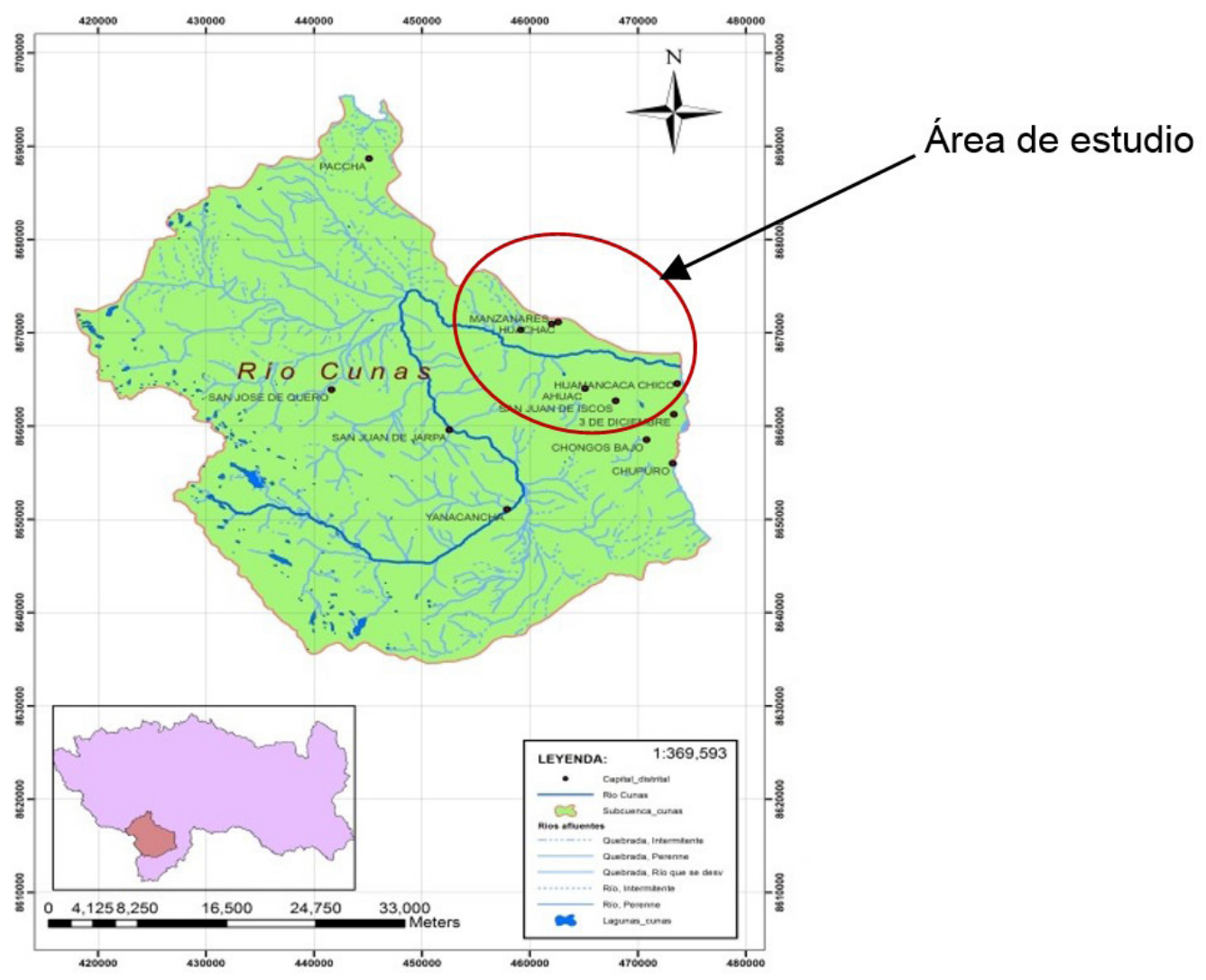

Figura 1. Ubicación del área de estudio en la subcuenca hidrográfica del río Cunas. Fuente: Municipalidad provincial de Chupaca (2011). 
El trabajo de campo se realizó en la primera quincena de abril del 2013, al finalizar la época de lluvias y en la primera quincena de julio, en los sectores de Angasmayo, Huarisca y Pilcomayo. Inicialmente, se definió las estaciones de muestreo en los sectores respectivos. La primera estación se ubicó a 10 metros aguas abajo del puente de Angasmayo, la segunda a 10 metros aguas abajo de la central hidroeléctrica de Huarisca y la tercera a 10 metros aguas abajo del puente Cunas.

\section{Recolección y análisis de las muestras de agua.}

\section{Análisis fisicoquímico del agua.}

Las muestras de agua fueron colectadas en dirección opuesta al flujo de la corriente, desde la superficie del río, en botellas de plástico de dos litros, previamente tratadas con una solución de ácido clorhídrico en proporciones iguales con agua destilada. Las muestras se refrigeraron a $4^{\circ} \mathrm{C}$ y fueron trasladadas al Laboratorio de Investigación de Agua de la Universidad Nacional del Centro del Perú para su posterior análisis dentro de los tiempos exigidos por la Normativa para cada parámetro.

Los parámetros determinados in situ fueron: oxígeno disuelto $(\mathrm{mg} / \mathrm{l})$, sólidos totales disueltos $(\mathrm{mg} / \mathrm{l})$, conductividad $(\mu \mathrm{S} / \mathrm{cm})$, temperatura $\left({ }^{\circ} \mathrm{C}\right), \mathrm{pH}$, turbidez (FTU). Estas mediciones se realizaron con equipos portátiles Hanna Instruments.

\section{Análisis bacteriológico del agua}

Las muestras fueron colectadas en frascos de vidrio estériles y refrigeradas hasta el momento de su análisis. La determinación de coliformes termotolerantes se realizó en el laboratorio de microbiología de la Facultad de Zootecnia de la Universidad nacional del Centro del Perú, según el método del Número más probable.

El Índice de calidad del agua (ICA), se calculó mediante la aplicación del INSF, para lo cual se utilizó la siguiente fórmula (5):

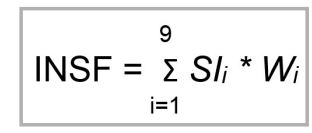

Dónde:

ISNF = Índice de la Fundación Nacional de Saneamiento de los Estados Unidos

$\mathrm{SI}_{\mathrm{i}}=$ Subíndice del parámetro i

$\mathrm{W}_{\mathrm{i}}=$ Factor de ponderación para el subíndice $\mathrm{i}$

El resultado final, se interpreta de acuerdo con la escala de calificación: excelente, buena, regular, mala y muy mala.

\section{Recolección y análisis de las muestras de macroinvertebrados bentónicos}

En cada sector de muestreo se definió un tramo de 10 metros. Las muestras de macroinvertebrados bentónicos fueron colectadas mediante una red Surber de 0,09 m2 de área de superficie de muestreo y una malla de abertura de $250 \mu \mathrm{m}$ de abertura. Cada muestra estuvo constituida por 5 réplicas. Las muestras fueron trasladadas al Laboratorio de Investigación de Agua en bolsa de polietileno gruesas, etiquetadas y fijadas con alcohol de $70^{\circ}$ para su respectiva identificación taxonómica. El índice biótico EPT, se calculó en base a tres órdenes de macroinvertebrados bentónicos, Ephemeroptera, Plecoptera y Trichoptera, sobre el total de individuos (6).

\section{RESULTADOS}

\section{Caracterización de la calidad del agua mediante el INSF}

El Índice de calidad de agua para cada sector y época de muestreo fue obtenido a partir de los indicadores fisicoquímicos y bacteriológicos a través del índice de la Fundación Nacional de Saneamiento de los Estados Unidos de Norte América (INSF).

El índice NSF en el sector de Angasmayo para la época de lluvia fue de 75,14, en Huarisca de 67,96 y en Pilcomayo de 61,39. En tanto que para la época de estiaje el INSF en Angasmayo fue de 74,23, en Huarisca de 65,22 y en Pilcomayo de 55,82. Los valores del INSF obtenidos en el tramo del río evaluado, muestran variación de la calidad de agua en los tres sectores de muestreo (Tabla 1).

El sector de Angasmayo, presentó una calidad de agua buena en época de lluvia y estiaje, lo cual indica que la calidad del agua está protegida, sólo existe un menor grado de amenaza y rara 
vez se aparta de las condiciones físicas o parámetros establecidos. En los sectores de Huarisca y Pilcomayo, presentaron una calidad de agua media en ambas épocas, lo cual revela que la calidad del agua suele estar protegida, pero se ve amenazada de vez en cuando y ésta se aparta de las condiciones físicas o parámetros establecidos.

Tabla 1. Calidad de agua del río Cunas mediante el índice de la Fundación Nacional de Saneamiento de los Estados Unidos, según sector y época de muestreo.

\begin{tabular}{|c|c|c|c|c|c|c|c|}
\hline \multirow[t]{2}{*}{ Indicador } & \multirow[t]{2}{*}{ Unidades } & \multicolumn{2}{|c|}{ Angasmayo } & \multicolumn{2}{|c|}{ Huarisca } & \multicolumn{2}{|c|}{ Pilcomayo } \\
\hline & & Lluvia & Estiaje & Lluvia & Estiaje & Lluvia & Estiaje \\
\hline Oxígeno disuelto & $\mathrm{mg} / \mathrm{l}$ & 14,28 & 14,79 & 12,75 & 12,07 & 10,88 & 7,82 \\
\hline $\begin{array}{l}\text { Coliformes termoto- } \\
\text { lerantes }\end{array}$ & $\mathrm{NMP} / 100 \mathrm{ml}$ & 8,4 & 8,4 & 7,05 & 5,55 & 4,35 & 3,00 \\
\hline $\mathrm{Ph}$ & Unidad & 10,8 & 11,16 & 10,56 & 10,8 & 11,04 & 10,56 \\
\hline $\mathrm{DBO}_{5}$ & $\mathrm{mg} / \mathrm{l}$ & 5,5 & 5,5 & 4,1 & 3,8 & 3,2 & 2,9 \\
\hline Temperatura & ${ }^{\circ} \mathrm{C}$ & 2,8 & 2,5 & 2,7 & 2,4 & 2,6 & 2,1 \\
\hline Fosfatos & $\mathrm{mg} / \mathrm{l}$ & 10 & 9,9 & 10 & 9,9 & 9,5 & 9,8 \\
\hline Nitratos & $\mathrm{mg} / \mathrm{l}$ & 10 & 9,9 & 10 & 9,9 & 9,9 & 9,8 \\
\hline Turbidez & FTU & 6,48 & 7,28 & 5,6 & 6,8 & 5,2 & 6,16 \\
\hline $\begin{array}{l}\text { Sólidos totales di- } \\
\text { sueltos }\end{array}$ & $\mathrm{mg} / \mathrm{l}$ & 6,88 & 4,8 & 5,2 & 0 & 4,72 & 3,68 \\
\hline Índice NSF & & 75,14 & 74,23 & 67,96 & 65,22 & 61,39 & 55,82 \\
\hline
\end{tabular}

\section{Caracterización de la calidad del agua mediante el índice biótico EPT}

Un total de 26 familias de macroinvertebrados bentónicos fueron colectadas en las tres estaciones de muestreo del río Cunas, durante las épocas de lluvia y estiaje. La clase Insecta fue la más representativa en abundancia y riqueza de taxa.

En la Tabla 2, se presentan los valores de la abundancia total de macroinvertebrados bentónicos y la abundancia de individuos de los órdenes Ephemeroptera, Plecoptera y Trichoptera. Los efemerópteros estuvieron representados por las familias Baetidae y Leptophlebiidae, los plecópteros por Perlidae y Gripopterygidae y, los tricópteros por Hydropsychidae, Hydrobiosidae, Hydroptylidae, Leptoceridae y Limnephilidae. La familia más abundante tanto en época de lluvia como de estiaje correspondió a Baetidae. 
Tabla 2. Abundancia de macroinvertebrados bentónicos del río Cunas, según sector y época de muestreo.

\begin{tabular}{|c|c|c|c|c|c|c|}
\hline \multirow[b]{2}{*}{ Familias } & \multicolumn{2}{|c|}{ Angasmayo } & \multicolumn{2}{|c|}{ Huarisca } & \multicolumn{2}{|c|}{ Pilcomayo } \\
\hline & Lluvia & Estiaje & Lluvia & Estiaje & Lluvia & Estiaje \\
\hline Baetidade & 607 & 903 & 826 & 604 & 103 & 108 \\
\hline Leptophlebiidae & 155 & 311 & 71 & 132 & 13 & 4 \\
\hline Perlidae & 183 & 205 & 12 & 56 & 0 & 0 \\
\hline Gripopterygidae & 210 & 310 & 55 & 65 & 0 & 0 \\
\hline Hydropsychidae & 221 & 171 & 101 & 78 & 7 & 0 \\
\hline Hydrobiosidae & 15 & 32 & 10 & 11 & 0 & 0 \\
\hline Hydroptylidae & 9 & 21 & 15 & 5 & 0 & 0 \\
\hline Leptoceridae & 65 & 73 & 38 & 37 & 0 & 0 \\
\hline Limnephilidae & 4 & 55 & 3 & 18 & 7 & 0 \\
\hline Abundancia de EPT & 1469 & 1581 & 1131 & 1006 & 130 & 112 \\
\hline Abundancia total & 2540 & 3026 & 3996 & 3780 & 5219 & 4207 \\
\hline
\end{tabular}

El índice EPT en el sector de Angasmayo fue de $57,83 \%$, en Huarisca de $28,30 \%$ y en Pilcomayo fue de 2,49\%, durante la época de lluvia (Figura 1). Mientras que en los sectores de Angasmayo, Huarisca y Pilcomayo el índice EPT fue de $52,25 \%$, $26,61 \%$ y $2,66 \%$, respectivamente, durante la época de estiaje.

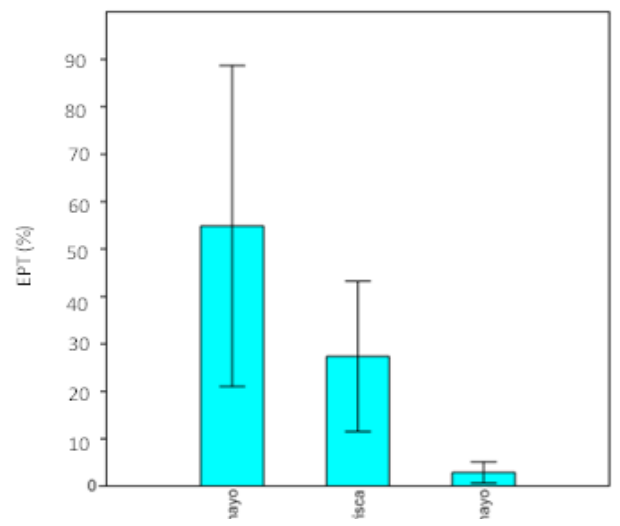

Figura 1. Calidad de agua del río Cunas según índice EPT.
$\%$, durante la época de lluvia (Figura 1). Mientras Las clases de calidad del agua obtenidas mediante el índice EPT en el tramo del río evaluado durante las dos épocas de muestreo fueron: clase 2 en Angasmayo, clase 3 en Huarisca y clase 4 en Pilcomayo. Estos resultados indican una variación de la calidad de agua en los tres sectores de muestreo. El sector de Angasmayo presentó una buena calidad de agua, lo cual revela que está levemente impactada. El sector de Huarisca presentó una regular calidad de agua, lo cual muestra que está siendo moderadamente impactada. En tanto, el sector de Pilcomayo presentó una mala calidad de agua, lo cual revela que la biodiversidad estaría siendo severamente impactada.

\section{DISCUSIÓN}

\section{Caracterización de la calidad del agua mediante el INSF}

El índice de calidad de agua es una herramienta muy útil para comunicar información sobre la calidad del agua a las autoridades y al público (1). Puede darnos rápidamente una imagen 
general del estado del recurso. Es muy útil para propósitos comparativos, y determinar qué puntos de muestreo muestran peor calidad de agua. Cualquier índice, por su diseño, contiene menos información que los datos a partir del cual se obtiene y del que constituye un resumen, por tanto no puede reemplazar el análisis detallado de los datos obtenidos a partir de un plan de monitoreo. Tampoco debe usarse como único criterio para la toma de decisión y el manejo de los recursos hídricos (5).

El índice de calidad de agua de la Fundación Nacional de Saneamiento de los Estados Unidos de Norte América (INSF) adopta para condiciones óptimas un valor máximo determinado de 100, que va disminuyendo con el aumento de la contaminación el curso de agua en estudio.

Las aguas con INSF mayor que 91, son aguas de categoría excelente, capaces de poseer una alta diversidad biológica y conveniente para todas las formas de contacto directo con ella. Las aguas con INSF mayor que 71, son aguas de categoría buena, poseen una menor diversidad biológica y el agua no es conveniente para todas las formas de uso. Mientras que las aguas con un INSF de categoría media, tienen generalmente menos diversidad biológica y han aumentado con frecuencia el crecimiento de las algas (3).

Las aguas con un INSF de categoría mala, presentan una baja diversidad de la vida acuática y están experimentando probablemente problemas de contaminación. En tanto que, las aguas con un INSF de categoría muy mala, pueden sólo poseer un número limitado de las formas de vida acuática, presentan numerosos problemas y normalmente no son aconsejables para las actividades que implican el contacto directo con ella, tal como natación $(6,7)$.

Los resultados obtenidos del índice de calidad de agua NSF en el sector de Angasmayo, en épocas de lluvia y estiaje revelan que las aguas presentan una calidad de agua buena, lo cual muestra que el grado de amenaza es menor, pues las condiciones son cercanas a las naturales. En los sectores de Huarisca y Pilcomayo, las aguas presentan calidad de agua media, lo cual indica que las condiciones en pocas veces son cercanas a las naturales y algunos de los usos pueden darse.

La variación de índice de calidad de agua NSF en los tres sectores de muestreo fue de buena en el sector de Angasmayo a media en el sector de Huarisca y Pilcomayo, las cuales presentan un ligero deterioro como consecuencia de las actividades antrópicas acentuadas en esta parte del río.

Estos resultados demuestran que las aguas del río Cunas, aún no experimentan severos problemas de contaminación, considerando que las aguas de calidad buena pueden soportar una importante diversidad de vida acuática y son apropiadas para todo tipo de recreación y para la toma de agua para potabilización. Sin embargo, las aguas de calidad media generalmente poseen menor diversidad de organismos acuáticos y frecuentemente manifiestan un crecimiento anormal de algas. Por tanto, si las actividades humanas continúan su desarrollo sin tener en cuenta los criterios ambientales la calidad del agua se verá seriamente afectada.

\section{Caracterización de la calidad del agua mediante el índice biótico EPT}

Los resultados obtenidos muestran una primera aproximación de evaluación de la calidad de agua a través de las comunidades de macroinvertebrados bentónicos en la subcuenca hidrográfica del río Cunas. De las 26 familias identificadas en el área de estudio sólo se utilizaron nueve para determinar el índice EPT.

La mayor abundancia de los individuos del orden Ephemeroptera se registró en Angasmayo. Resultados que son corroborados por Rivera et al. $(8,9)$ quienes refieren que la gran mayoría de las especies de este orden poseen baja tolerancia a la contaminación. Pues prefieren vivir en sitios con buena oxigenación, en sustratos de piedra y arena. La familia con mayor abundancia fue Baetidae, los individuos de esta familia pueden tolerar cierto grado de contaminación (10). Ello explica la presencia de Baetidae en el sector de Pilcomayo.

El orden Plecoptera con las familias Perlidae y Gripopterygidae, está restringido al sector de Angasmayo, principalmente. Los resultados son respaldados por Molina et al. (11), quienes refieren que los individuos de este orden son los más exigentes dentro del grupo de los macroinvertebrados en cuanto a calidad de agua.

El orden Trichoptera constituye un importante Páginas 98-105 
componente de las comunidades bénticas y base de la cadena trófica de los ecosistemas acuáticos (9). La mayor abundancia del orden Trichoptera fue registrada en el sector de Angasmayo, en donde la calidad del agua es buena. La familia más representativa por su abundancia es Hydrosychidae. Estos resultados son corroborados por Acosta (12) y Rodríguez et al. (13), quiénes señalan que los tricópteros son indicadores de aguas limpias y su abundancia aumenta con el aumento de la altitud.

La calidad de agua del río Cunas en el sector de Angasmayo obtenida mediante el ICA - EPT, revela el bajo nivel de contaminación orgánica que presenta. Sin embargo, aguas abajo se incrementa la contaminación por la evacuación de aguas residuales al curso del río, la cual se ve reflejada por el descenso de la abundancia de la entomofauna bentónica, principalmente de los EPT.

El estudio se llegó a las siguientes conclusiones:

1. La calidad del agua del río Cunas obtenida mediante el índice de calidad de agua ICA-NSF, según sector y época de muestreo fue:

- En Angasmayo, la calidad de agua tanto en época de lluvia como en estiaje calificó como agua de calidad buena.

- En los sectores de Huarisca y Pilcomayo, la calidad de agua en ambas épocas de muestreo calificó como agua de calidad media.

2. La calidad del agua del río Cunas obtenida mediante el índice de calidad de agua EPT, según sector y época de muestreo fue:

- En Angasmayo, la calidad de agua tanto en época de lluvia como en estiaje calificó como agua de calidad buena (categoría 2).

- En Huarisca la calidad de agua tanto en época de lluvia como en estiaje calificó como agua de calidad regular (categoría 3).

- En Pilcomayo, la calidad de agua tanto en época de lluvia como en estiaje calificó como agua de calidad mala (categoría 4).

\section{REFERENCIAS}

Pérez CA, Rodríguez A. (2008). Índice fisicoquímico de la calidad de agua para el manejo de lagunas tropicales de inundación. Rev. Biol. Trop. 56(4): 1905-1918.

Figueroa R, Valdovinos C, Araya E, Parra O. (2003). Macroinvertebrados bentónicos como indicadores de calidad de agua de los ríos del Sur de Chile. Revista Chilena de Historia Natural. 76: 275-285.

Segnini S. (2003). El uso de macroinvertebrados bentónicos como indicadores de la condición ecológica de los cuerpos de agua corriente. Ecotrópicos. 16(2): 45-63.

Custodio, V.M. y Pantoja, E.R. (2012). Impactos antropogénicos en la calidad del agua del río Cunas. Rev. Apunt. cienc. soc. ; 02(02): 130-137.

Valcárcel, R. L., Macías, A. N., Fonseca, F. D. (2009). El Índice de Calidad de Agua como herramienta para la gestión de los recursos hídricos. Cuba: Medio Ambiente y Desarrollo; Revista electrónica de la Agencia de Medio Ambiente. 16: 1-5.

Fernández, H.R., Romero F, Vece, M.B., Manzo V., Nieto, C. y Orce, M. (2002).Evaluación de tres índices bióticos en un río subtropical de montaña (Tucumán- Argentina). Limnetica. 21 (1-2): 10-13.

Castillo, A. y Sánchez, D.L. (2004) .Estudio de la calidad de las aguas de la Vega de Granada. Aplicación al riego del tabaco. Consejería de Agricultura y Pesca de la Junta de Andalucía.

Alonso, F. A. (2005) . Valoración de la degradación ambiental y efectos ecotoxicológicos sobre la comunidad de macroinvertebrados bentónicos en la cabecera del río Henares [Tesis para optar el grado académico de Doctor]. Alcalá: Universidad de Alcalá.

Rivera, U.J., Camacho, P.D. y Botero, B.A., (2008). Estructura numérica de la entomofauna acuática en ocho quebradas del Departamento del Quindío-Colombia. Acta biol. Colomb.13 (2): 133-146.

Romero, B., Pérez, S., y Rincon, M. (2006). Ephemeroptera del Parque Nacional Natural "Cueva de los Guácharos" Huila-Colombia. Rev. UDCA Actual. Divulg. Cient. 9(1), 141-149.

Molina, C., Gibon, F., Pinto J. y Rosales, C. (2008). Estructura de macroinvertebrados acuáticos en un río altoandino de la cordillera Real, Bolivia: variación anual y longitudinal en relación a factores ambientales. Ecología Aplicada, 7(1,2).

Acosta, R.C. (2009). Estudio de la cuenca altoandina del río Cañete (Perú): Distribución altitudinal de la comunidad de macroinvertebrados bentónicos y caracterización hidroquímica de sus cabeceras cárticas [Tesis para optar el grado académico de Doctor]. Barcelona: Universidad de Barcelona.

Rodríguez, E., López, M. y Betancourth, J. (2003). Construcción de la línea Base sobre el Estado de la Biodiversidad en Colombia. Bogotá: Instituto de Investigación de Recursos Biológicos Alexander von Humboldt. 